\title{
Errata: Segmentation of nucleus and cytoplasm of a single cell in three- dimensional tomogram using optical coherence tomography
}

Chia-Kai Chang

Chien-Chung Tsai

Wan-Yi Hsu

Jau-Shiuh Chen

Yi-Hua Liao

Yi-Shuan Sheen

Jin-Bon Hong

Ming-Yi Lin

Jeng-Wei Tjiu

Sheng-Lung Huang 


\title{
Errata: Segmentation of nucleus and cytoplasm of a single cell in three-dimensional tomogram using optical coherence tomography
}

\author{
Chia-Kai Chang, ${ }^{a}$ Chien-Chung Tsai, ${ }^{a}$ Wan-Yi Hsu, ${ }^{a}$ Jau-Shiuh Chen, ${ }^{b}$ Yi-Hua Liao, ${ }^{b}$ Yi-Shuan Sheen, ${ }^{b}$ \\ Jin-Bon Hong, ${ }^{b}$ Ming-Yi Lin, ${ }^{b}$ Jeng-Wei Tjiu, ${ }^{b}$ and Sheng-Lung Huang ${ }^{a}$ \\ ${ }^{a}$ National Taiwan University, Graduate Institute of Photonics and Optoelectronics, Taipei, Taiwan \\ ${ }^{b}$ National Taiwan University, Department of Dermatology, National Taiwan University Hospital and College of Medicine, Taipei, Taiwan
}

[DOI: 10.1117/1.JBO.22.3.039801]

This article [J. Biomed. Opt. 22(3), 036003 (2017)] was originally published online on 2 March 2017 with an error. In the last sentence on p. 3 , the noise level for $\Delta I=3$ was incorrectly defined as 0.977 . It should be 0.989 .

This article was corrected online on 7 March 2017. It appears correctly in print. 